\title{
Comment est née «Marianne»? La caricature, médiatrice de la figuration de la République en France
}

\author{
Annie Duprat \\ Professora de Historia Moderna na Université de Versailles Saint-Quentin-em-Yvelines e do Centre \\ d'Histoire Culturelle des Sociétés Contemporaines - CHCSC. Autora de, entre outros livros, Marie- \\ Antoniette. Une reine brisée. Paris: Perrin, 2006. annie.duprat@wanadoo.fr
}

\section{RESUMO}

"Marianne", um símbolo da cultura clássica, foi apropriado pela população francesa, tornandose a personificação da República. Esta figura feminina venceu Hércules nos embates dos símbolos, por melhor representar, não só a Liberdade, mas a noção de República. Esta pesquisa se centrou entre os anos de 1830, de Delacroix, e 1848, do concurso da figura da República, e se propôe a estudar como as caricaturas, comumente denunciadoras e destruidoras de imagens, foram importantes fontes mediadoras da manutenção da imagem da República como uma mulher.

PalAVRAS-ChAVE: "Marianne"; caricatura; República; França.

\section{ABSTRACt}

"Marianne", a symbol of classic culture, was appropriated by French people, becoming the personification of Republic. This feminine figure won Hercules in a symbolic dispute because it is a better representation, not only of Liberty, but of Republic notion. This research is centred during the 1830's, from Delacroix; and 1848, to the contest of Republic's figure, and proposes to study of how caricatures, usually denouncer and destroyer of images, were an important historical source for the maintenance of feminine figure as the image of Republic. KEY WORDS: "Marianne"; caricature; Republic; France. 


\section{Comment est née «Marianne»? La caricature, médiatrice de la figuration de la République en France}

En France, l'instauration d'un régime républicain s'est opéré de façon à la fois brutale et impromptue, quelques semaines après la prise des Tuileries le 10 août 1792, I'emprisonnement du roi Louis XVI le 14 août, la victoire de Valmy sur les troupes austroprussiennes le 20 septembre et l'abolition de la royauté le 21 . Sans être à proprement dire «proclamée», la République s'est installée de fait, dans les mots avant de l'être dans les actes législatifs. En effet, on peut lire en octobre 1792 dans La Feuille Villageoise, un journal destiné aux habitants des campagnes, l'observation suivante, à propos du décret mettant en place une nouvelle assemblée, la Convention nationale:

«Le 21 septembre, la Convention nationale s'est constituée... elle est assemblée dans une crise révolutionnaire ; elle est assemblée sans la présence du roi ; elle est assemblée pour former un plan nouveau de gouvernement [...]. Le président a déclaré qu'il faut abolir la race funeste des rois [...] qui sont dans l'ordre moral ce que les monstres sont dans l'ordre physique [...]. Les cours sont l'atelier des crimes [...]. Le président a mis aux voix ce décret, adopté unanimement: la Convention décrète que la royauté est abolie en France. D'après cette résolution, il a été décrété qu'à l'avenir, toutes les lois et tous les actes publics seraient datés de l'an premier de la République ${ }^{1} \ldots$...
La raison d'une telle impréparation peut être trouvée dans le fait qu'un an plus tôt, lors de la tentative de fuite du roi hors des frontières du royaume, l'idée même d'une République avait été rejetée ${ }^{2}$. En effet, les esprits de l'époque considéraient qu'un régime républicain ne pouvait fonctionner correctement que dans un petit pays comme les Provinces-Unies ou dans le cadre d'une cité-état comme l'était Genève. L'établissement d'une République aux EtatsUnis en 1776 ne les avait pas encore convaincus car ce système était fondé sur une base fédéraliste. Dans le même ordre d'idées, aucune réflexion concernant la création d'un signe permettant de l'identifier n'a eu lieu. On cite souvent la proposition faite par les députés Camus et Grégoire, le 15 août 1792, d'un sceau destiné aux Archives Nationales comme étant la première image de la République française; pourtant, il n'en est rien, même si la figure ainsi choisie peut être considérée comme la matrice de toutes les suivantes car elle est directement inspirée par la figure de la Liberté telle qu'elle avait été établie à l'époque de la Renaissance par les auteurs d'allégories et d'emblèmes comme le plus fameux d'entre eux, I'italien Cesare $\mathrm{Ripa}^{3}$. On y reviendra plus loin.

\footnotetext{
1 La Feuille villageoise, numéro 51, octobre 1792. Voir Melvin Edelstein, La Feuille villageoise. Communication et modernisation dans les régions rurales pendant la Révolution, Paris, Bibliothèque Nationale, 1977.

2 Timothy Tackett, Le roi s'enfuit. Varennes et l'origine de la Terreur, Paris, La Découverte, 2004.

${ }^{3}$ Cesare Ripa, Iconologie ou Explication nouvelle de plusieurs images, emblèmes et autres figures hyérogliphiques des vertus, des vices, des arts, des sciences... Tirée des recherches et des figures de César Ripa, desseignées et gravées par Jacques de Bie et moralisées par J. Baudoin, Paris, Villery, 1637
} 


\section{Une allégorie classique: la Liberté}

L'allégorie de la Liberté a traversé les pays et les siècles au rythme de la diffusion des traductions de Ripa en Europe comme dans l'Amérique coloniale. Une quinzaine d'années avant la Révolution française, deux artistes, Charles-Nicolas Cochin et Charles Gravelot lancent en 1765 un Almanach iconologique annuel dans lequel figure encore une allégorie de la Liberté sous la forme d'une femme vêtue à l'antique et coiffée du bonnet phrygien. Le signe est donc bien établi à partir de 1789; il se retrouve souvent sur les vignettes des sociétés populaires ou des nouvelles municipalités, sur les drapeaux des sections ou des bataillons comme on peut le voir sur les estampes de Vieilh de Varennes mais il n'identifie pas précisément ce régime nouveau qu'est la République ${ }^{4}$.

La question de la représentation du pouvoir dans un régime démocratique, par essence abstrait puisque fondé sur l'ensemble de la communauté de citoyens acceptant un certain ombre de valeurs communes, est délicate et I'histoire de l'élaboration de la figure de celle que les Français nommeront par la suite familièrement «Marianne» mérite d'être reprise pour mieux comprendre comment un signe allégorique relevant de la culture classique de l'élite a pu s'être approprié par le plus grand nombre au point de devenir familier. Le passage de la monarchie, qui part du principe de l'obéissance des sujets à un souverain héréditaire de droit divin, à la République, fondée sur la primauté de la Loi, ce qui suppose une relation de confiance entre les citoyens et leurs représentants, a de nombreuses conséquences, en particulier dans le domaine de la représentation. Tandis que la monarchie s'incarne dans la personne du roi et trouve matière à s'identifier dans le récit des cérémonies (mariages, obsèques, entrées royales, imagerie plus familière de la famille royale) et des hauts faits du prince (batailles, conquête de territoires ou signatures de traités) les bases mêmes sur lesquelles est fondée la République sont abstraites puisqu'elles ne sont pas incarnées dans une personne (le corps du roi) mais dans l'acceptation par le corps des citoyens d'un certain nombre de valeurs.

Si le vocabulaire des iconologies, ces dictionnaires des signes à destination des artistes, s'épuise progressivement au cours du siècle des Lumières, il reste encore présent à la mémoire des contemporains et la dernière grande iconologie, lancée par Charles-Nicolas Cochin et Charles Gravelot, I'Almanach iconologique, rencontre un grand succès; sa publication s'étend jusqu'en 1781 et constitue 17 volumes $^{5}$. A la mort de Gravelot en 1773, Cochin reprend le flambeau et la publication se poursuit jusqu'en 1781; il préparait une refonte totale de l'ouvrage lorsqu'il disparaît à son tour en 1790, au moment même de l'explosion révolutionnaire. Le livre est à nouveau édité à Paris chez le libraire Lattré rue Saint-Jacques en 1791 sous le titre Iconologie par figures ou traité complet des allégories, emblèmes etc. ouvrage utile aux artistes, aux amateurs et peuvent servir à l'éducation des jeunes personnes, assorti d'un «discours préliminaire» qui est à la fois un bref historique du genre de l'iconologie et une défense de la permanence des signes; on lit en effet qu'il faut éviter «le néologisme, ou l'abus des nouveaux emblèmes». Le registre

\footnotetext{
${ }^{4}$ Vieilh de Varenne, Description curieuse et intéressante des soixante drapeaux que l'amour patriotique a offerts aux soixante districts de la ville et faubourgs de Paris... Paris, Sorin, 1790.

${ }^{5}$ Gravelot (Charles) et Cochin (Charles-Nicolas), Iconologie par figures ou traité complet des allégories, emblèmes etc. ouvrage utile aux artistes, aux amateurs et peuvent servir à l'éducation des jeunes personnes, Paris, Lattré, rééd. 1791.
} 
des figures de l'iconologie de Gravelot et Cochin est toujours très classique et les commentaires explicatifs très sobres.

Dans cette édition, on retrouve la classique «Liberté» (figure 1, à gauche) une femme:

«tenant d'une main un sceptre et de l'autre un bonnet. Le sceptre exprime l'empire que par elle l'homme a sur lui-même. [A ses pieds] le chat, ennemi de la contrainte, achève de caractériser la Liberté». La description insiste également sur la présence des bateaux, au fond de l'image, et des oiseaux dans les airs «qui changent de climat avec les saisons».

La Liberté est aussi «la mère des connaissances» ce qui explique la présence de livres, d'une palette, d'une lyre, d'un compas et d'un bouclier qui rappellent les arts, les sciences et la guerre.

Sous le titre «La Liberté» une nouvelle gravure est réalisée par le fils de CharlesNicolas Cochin (figure 1, à droite) qui présente des changements significatifs parce qu'ils sont clairement en relation avec les événements révolutionnaires qui viennent de se dérouler: le bonnet est posé sur une pique, la jeune femme foule aux pieds un joug brisé, tandis que derrière elle se tient:

"la licence, jeune femme nue, échevelée, ayant une couronne de vigne sur sa tête. La couronne

Doc 27a et 27b. ICOÑOLOGIES DE LA LIBERTÉ
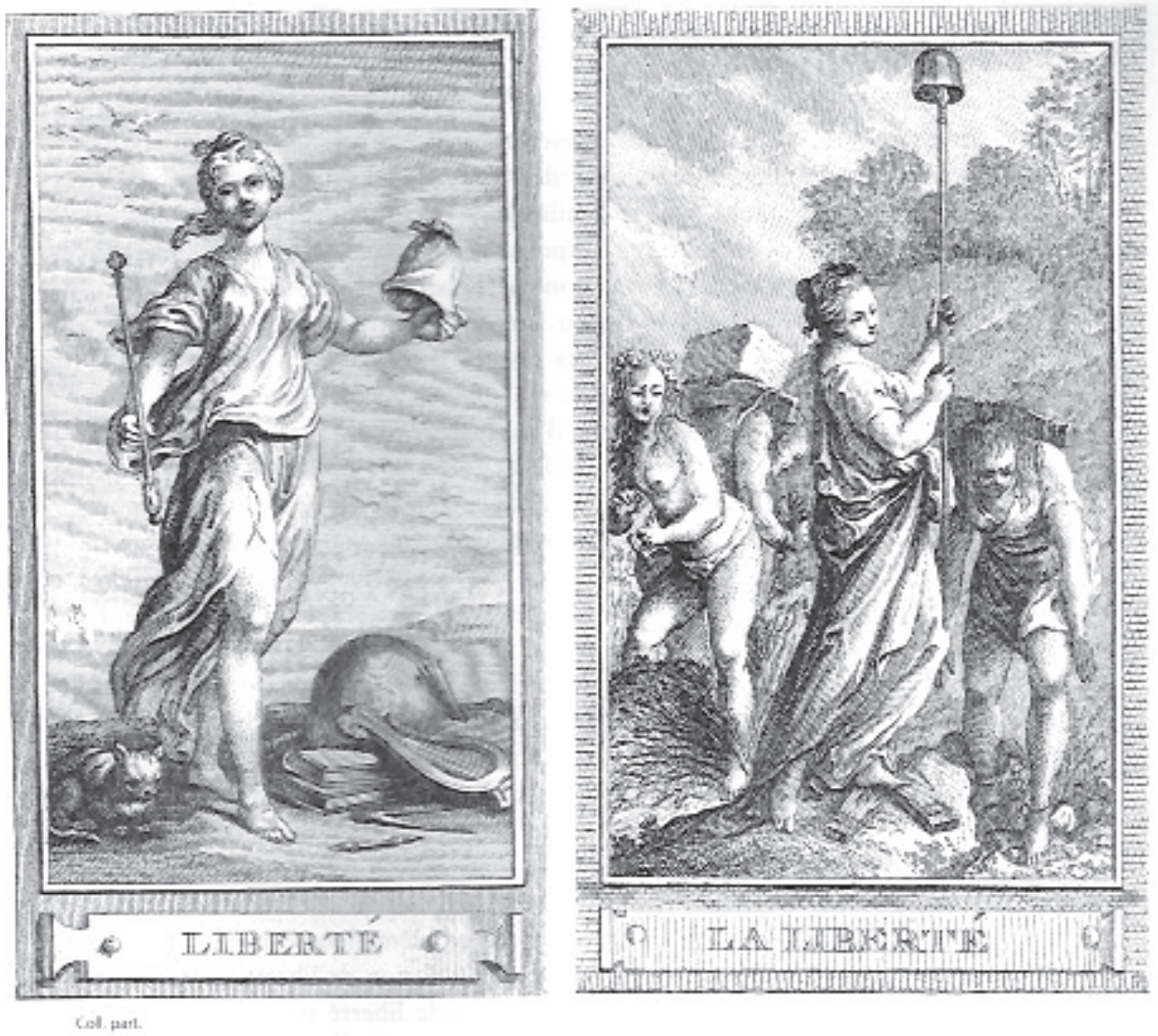

Figure 1. Liberté par Gravelot et Cochin 
de vigne est relative aux excès où se portaient les bacchantes. La licence brise le frein de la raison, traverse, foule aux pieds un champ de blé et franchit la borne et la haie quil'entoure».

Au premier plan à droite, les pieds entravés et le dos courbé, une femme ressemble à la figure de «l'esclavage» dont il est question plus loin dans la notice. On mesure l'impact de la Révolution entre la première figure, qui utilise sobrement une emblématique conventionnelle et la seconde, qui fait vivre en quelque sorte la notion de liberté, en montrant par le jeu du bonnet posé sur la pique que la liberté se conquiert, par la violence si nécessaire, mais que, tout en préservant de «l'esclavage», elle doit éviter «la licence».

Au cours de la période révolutionnaire, de nombreuses allégories de la Liberté sont produites sur ce modèle; lorsqu'on y voit le triangle de l'Egalité, le faisceau des licteurs (pour le pouvoir de la Loi), la présence d'enfants (souvent un enfant blanc et un enfant noir associés pour la Fraternité), on peut estimer qu'il s'agit d'une allégorie de la République. En effet, s'il n'y a pas eu de concours artistique pour élaborer une figure officielle de la République, on a vu que dès le mois de septembre 1792, sur proposition du député Camus et de l'abbé Grégoire, un sceau spécifique pour les Archives Nationales avait été créé; il représente une femme débout, la main droite posée sur un faisceau de licteurs (le pouvoir de la Loi), la main gauche tenant une pique coiffée d'un bonnet de la Liberté. Entre 1789 et 1799 , près des $2 / 3$ des représentations de la République, sur les journaux, les correspondances officielles ou les affiches, proviennent de cette image sans qu'une norme n'ait été imposée. Durant I'an II (1794), un combat des symboles est livré entre les partisans d'un régime révolutionnaire autoritaire qui souhaitent imposer Hercule (ce demi-dieu antique étant investi de toute la force la force populaire, ce qui appartient au registre proprement révolutionnaire) et ceux qui sont partisans de l'allégorie féminine traditionnelle de la Liberté (ce registre est plus traditionnel car il renvoie également à l'iconographie mariale, très forte dans un pays catholique). Ce choix primitif, par défaut en quelque sorte, associe durablement l'idée de Liberté à celle d'une République féminine; malgré tous les efforts des patriotes les plus radicaux pour imposer la figure d'Hercule comme signifiant du Peuple, la Révolution et/ou la République s'efface derrière celle d'une femme. Cette observation, qui se fonde sur des pré-supposés politiques (la radicalité d'une Révolution jacobine qui se retrouverait dans la figure d'Hercule face à la modération d'une Révolution illustrée par la figure féminine) a conduit un certain nombre d'historiens, en particulier du «gender» nord-américain, a en tirer des conclusions faussées parce qu'elle ne prennent pas en compte le rythme de longue durée des représentations allégoriques ${ }^{6}$. On peut facilement démontrer que dans tous les décors et les projets étudiés, la figure traditionnelle de la Liberté (donc une figure féminine) s'est d'abord imposée, sans jamais perdre du terrain vis à vis de la figure masculine d'Hercule?.

\footnotetext{
${ }^{6}$ Lynn Hunt, Politics culture and class in the French Revolution, Berkeley, 1984; id. « Pourquoi la République est-elle une femme ? La symbolique républicaine et l'opposition des genres ", dans Révolution et République. L'exception française, Kimé, 1994, p. 358365 ; Antoine De Baecque, "The allegorical image of France, 1750-1800 : a political crisis of representation », Representations 47, summer 1994, p. 111-145.

7 Annie Jourdan, "L'allégorie révolutionnaire. De la Liberté à la République ", XVIIlème siècle, n³7, 1995, p. 503-532; id. "Libertés du XVIIIè siècle : concepts et images ", Visualisation, concepts et symboles du XVIIIè siècle européen, ss dir. Roland Mortier, Berlin, Arno Spitz, 1999, p. 39-58; id. Les monuments de la Révolution. Une histoire de représentation, Paris, Champion, 1997.
} 
La lère République, qui n'a pas eu de signe officiel d'identification, a également vécu une histoire aussi brève que tempétueuse, entre septembre 1792 et novembre 1799 (le coup d'Etat de Napoléon au 18 brumaire). Le mot de «République» comme les signes qui y renvoient sont alors interdits et désertent I'espace public. Lorsque la surgit Révolution de 1830, le tableau La liberté guidant le peuple par Eugène Delacroix rencontre un succès immédiat sans doute parce que le peuple a retrouvé dans cette figure féminine escaladant les barricades le souvenir des multiples «libertés» qui occupaient l'espace public de la France révolutionnaire. Mais le règne de Louis-Philippe ler n'est pas un régime républicain et, à nouveau, le mot est proscrit même si le signe, par l'intermédiaire de l'art de la caricature, continue à exister.

\section{L'apport d'un genre populaire: la caricature}

Le XIXè siècle a été qualifié d'âge d'or de la caricature, non seulement parce que de très grands talents se sont déployés dans la presse française illustrée, mais aussi parce que les années qui séparent la fin de l'Empire de I'installation définitive de la République ont été fertiles en rebondissements politiques et en scandales de toute nature, sources d'inspirations constamment renouvelées. Mais ces caricatures, qui nous permettent de poser un regard distancié sur l'histoire, peuvent également nous aider à comprendre comment les allégories de la Liberté, qui appartiennent au genre savant de I'iconographie classique, ont été en quelque sorte adoptées par les dessinateurs pour figurer, au delà de la Liberté, la notion de République. La caricature va jouer un rôle important sous le règne de Louis-Philippe (1830-1848) dans l'adoption d'une figure féminine quasiment charnelle comme figure de la République. Notons également que la multiplication des fêtes des rosières (les jeunes filles convenables) et le développement du catholicisme au féminin caractérisé par diverses apparitions mariales a pu contribuer à renforcer le lien qui, finalement, transformera l'allégorie féminine de la Liberté en une allégorie de la République plutôt bonne fille sous le nom de Marianne.

Mais pourquoi choisir d'observer les caricatures pour étudier les représentations politiques officielles? Parce que ces petites images vite lues et largement diffusées (en particulier par le journal de Philipon La Caricature) participent de la création d'imaginaires visuels, donc d'imaginaires mentaux. Devant être comprises rapidement, elles utilisent des codes déjà bien connus, mais qui n'ont pas de fixité sémantique, ce qui pose de multiples problèmes aux analystes qui cherchent souvent à montrer de façon théorique à quels dangers peuvent être confrontés les chercheurs; la caricature est l'expression d'un moment particulier de la vie des sociétés qui la génèrent. En effet, si représenter la République française sous l'image de Marianne nous apparaît évident, tel n'était pas le cas au cours du XIXè siècle comme en témoigne la trilogie de Maurice Agulhon (Marianne au combat, 1979 et Marianne au pouvoir, 1989 et Les métamorphoses de Marianne, 2001) Tel n'était pas non plus le cas durant la période révolutionnaire alors que tout va changer avec la Révolution de 1848. Pourtant, Maurice Agulhon n'envisage à aucun moment le rôle que les caricatures ont pu jouer dans la permanence du signe et son imprégnation culturelle. II ne fait que constater la permanence de l'union de sens entre la Liberté et la République lorsqu'il écrit dans Marianne au combat: 
"La Liberté (en son image féminine traditionnelle) a été présente en juillet 1830 ; mais elle portait avec elle le souvenir de son ancienne union, scellée dans les combats, avec la République. De la première de ces deux évidences il ressort que le nouveau régime ne pouvait pas éliminer entièrement cette effigie, et de la seconde qu'il ne l'admettrait pas sans réticences ${ }^{8} \%$.

La seconde République, proclamée en février 1848, entreprend d'ouvrir un concours de la figure de la République (peinture, sculpture, gravure, médailles etc...); ce concours remporte un grand succès chez les artistes si on en juge par le nombre d'œuvres envoyées même si le concours a échoué et n'a pas atteint ses objectifs.

Notre propos sera ici de rechercher comment, entre le 1830 de Delacroix et le 1848 du concours de la figure de la République, les caricatures utilisant des allégories de «femmes-Liberté» ou de «femmes-France» ou encore de «femmesRépublique» ont entretenu l'idée d'une figuration de la république comme une femme. La source la plus riche en ce domaine est le journal hebdomadaire La Caricature, fondé par Philipon en novembre 1830 et qui cesse de paraître en août 1835, en raison d'une loi de censure particulièrement brutale.

\section{Observer et compter}

Reste à identifier et à mesurer, année par année, la présence de ces «femmes-Liberté» que I'on peut aussi identifier comme des «femmes-République» dans plus de 500 planches (dans les 251 numéros du journal La Caricature parus entre le 4 novembre 1830 et le 27 août 1835).

Sur 58 figures, une jeune femme est associée au bonnet de la Liberté (soit qu'elle le porte sur la tête soit qu'il figure dans le champ de l'image), ce qui correspond à moins de $12 \%$ du total, et confirme l'idée qu'il n'y a pas d'obsession républicaine dans l'équipe de Philipon: dans l'iconographie et dans les textes de son journal, le souvenir de la Révolution demeure brouillé, obscurci par les préoccupations du moment. Parmi les années citées en référence, 1790 supplante 1789 autant que 1792-1794, mais le souvenir de la liberté dans un monde où tout paraissait possible mobilise les collaborateurs du journal, atterrés par la médiocrité du « système ». La mémoire des années 1830-1835 a donc choisi l'année de consolidation des acquis de la Révolution, 1790, au détriment de l'année du basculement, 1789, et de celles des déchirements, qui sont autant de déchirures, 1792-1794.

Considérons le flux de ces 58 figures année par année:

- en 1831, 11 figures mettent en scène une femme porteuse du bonnet et 5 figurent le bonnet seul ou comme élément du décor (environ $16 \%$ du total) ;

- en 1832, 14 planches retiennent notre attention. Sur 6 d'entre elles, les jeunes femmes sont coiffées du bonnet de la Liberté, tandis que sur 5 autres elles sont soit têtes nues, soit têtes coiffées de tours, signe afférent aux villes dans l'iconologie traditionnelle. Mais la caricature se permet de détourner les codes qu'elle utilise et l'image, comme la lettre qui accompagne ces cinq planches fait référence explicitement soit à la France, soit à la Liberté. Enfin, le bonnet de la Liberté est présent, seul, sur 3 lithographies (14\% du total);

- en 1833, 4 planches montrent des femmes coiffées du bonnet, 3 sont coiffées de tours et 5 planches présentent des bonnets seuls $(12 \%)$;

\footnotetext{
8 Maurice Agulhon, Marianne au combat. L'imagerie et la symbolique républicaines, Paris, 1979, p. 67.
} 
- en 1834, les jeunesfemmes, toujours coiffées, sont au nombre de 9 et le bonnet seul ne se rencontre que sur 2 planches (11\%);

- en 1835 enfin, année amputée qui ne voit la publication que de soixante-douze lithographies, on ne compte que 2 jeunes femmes coiffées du bonnet de la Liberté et 3 coiffées de tours figurant encore la France (7\%).

Un tel comptage minutieux sur un corpus assez modeste est important dans la mesure où il montre que le signe persiste, même si, progressivement, sa place se réduit (on passe d'environ $16 \%$ du total en 1831 , à $14 \%$ en 1832 , puis $12 \%$ en $1833,11 \%$ en 1834 et $7 \%$ en 1835). Devrait-on pour autant conclure à la disparition progressive des signes de la République dans les images du journal La Caricature ou y voir les effets conjugués de la censure et d'une actualité politique de plus en plus conflictuelle?

Tandis que la Restauration des Bourbons s'était accompagnée du retour aux figures traditionnelles du pouvoir royal, le règne de Louis-Philippe, né dans des circonstances particulières, rompt avec les représentations symboliques traditionnelles du pouvoir. La situation de la monarchie de Juillet est ambiguë et s'inscrit davantage dans le cadre du non-dit, de l'élision, voire de l'évitement, que dans celui de la représentation ostentatoire. Louis-Philippe, "roi des barricades», ne trouve pas aisément sa place dans un quelconque système de signes de représentations, et ceci soulève l'importante question non plus seulement de sa légitimité, mais de son existence même. Tandis que le nouveau régime cherche à construire son identité, en particulier par la peinture, la caricature occupe le territoire vide des imaginaires sociaux ${ }^{9}$. Les signes afférents à la Liberté et à la République, mécaniquement répétés et placés dans des séquences drolatiques, contribuent à occuper les vides de l'espace politique, à habituer l'œil, I'esprit, la conscience et la mémoire, à un nouvel imaginaire et donc à la possibilité d'un nouveau système politique: en bref, la caricature, de dénonciatrice et destructrice, se fait, à son insu, créatrice d'un nouvel imaginaire politique. Grâce aux caricatures, on peut étudier l'autre face de la représentation du pouvoir, l'image qui naît de la critique, de la dénonciation caustique, voire même de la calomnie, puisque telles sont les armes des journalistes et des dessinateurs satiriques: c'est l'image de la Liberté, de la République/Liberté et son association avec la France, France-République et France-Liberté, qui apparaissent dans la presse polémique et nous permettent de discerner «l'ombre portée» de la République de 1793 en $1830^{10}$.

Balzac, co-fondateur de La Silhouette avec Emile de Girardin, écrivait sur la caricature: "Cet art est une puissance ${ }^{11}$ ». Les événements qui se bousculent dès les premiers mois de la monarchie de Juillet stimulent l'ardeur des journalistes, et l'on trouve dans le journal $L a$ Caricature, des récits d'émeutes, d'insurrections réprimées et de barricades démantelées presque aussitôt que dressées. La presse dénonce alors par le texte et par l'image le triste état d'une France qui aspire à la liberté et les dessinateurs qui s'acharnent, par le dessin et par l'écrit, contre LouisPhilippe et son régime, car leur but est de déprécier cette monarchie du riflard, des pantoufles et des rodomontades inutiles; ils

\footnotetext{
${ }_{9}$ Michaël Marrinan, Painting politics for Louis-Philippe : art and ideology in Orleanist France, 1830-1848, New Haven, Yale University press, 1988; Bronislaw Baczko, Les imaginaires sociaux, mémoires et espoirs collectives, Paris, Payot, 1984.

${ }_{10}$ Lettre d'une planche de Grandville en 1830.

${ }^{11}$ Cité par Antoinette Huon, «Philipon et la maison Aubert », in Etudes de Presse, vol. 9, n 17, 4, pp. 67-76.
} 
ne peuvent de fait militer ouvertement en faveur d'une République dont les contours politiques sont loin d'être clairs à leurs yeux, comme en témoigne Le carnaval politique de Grandville et Forest ${ }^{12}$.

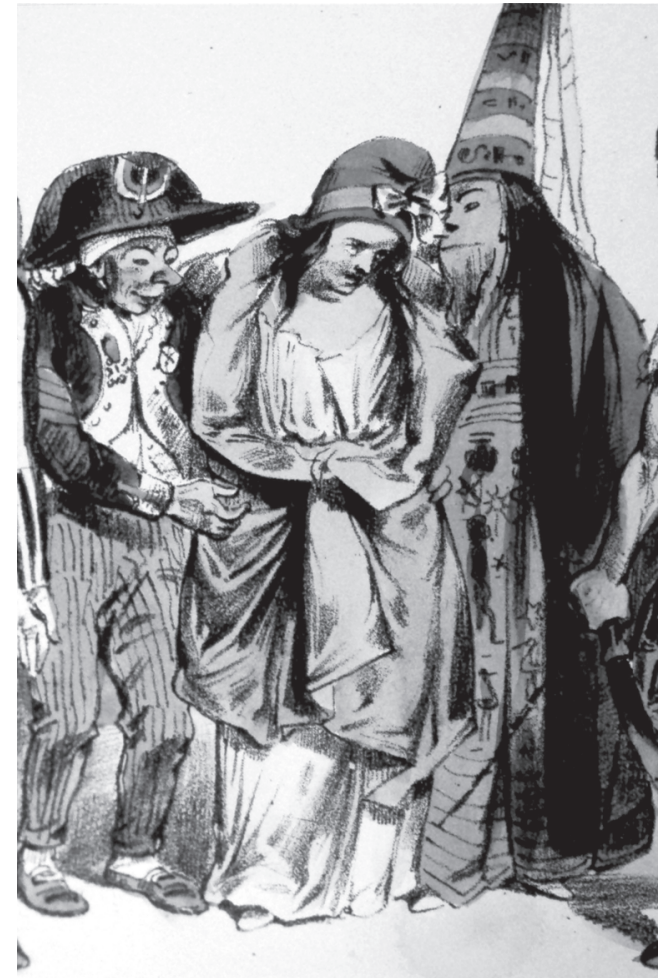

Figure 2. Carnaval politique, par Grandville et Forest, dans La Caricature, 10 mars 1831

Sur la première procession (figure 2), la malheureuse femme-liberté porteuse du bonnet phrygien est prisonnière $d u$ Bonhomme Carnaval, tandis que sur la seconde, une image dans l'image sous la forme d'un petit tableau discrètement accroché au mur, figure une «République fantôme de cauchemar», qui porte un bonnet, mais tient une hache et une tête coupée (figure 3). On ne saurait mieux résumer le lancinant débat sur le lien entre Terreur et Révolution et la nature de la Terreur.

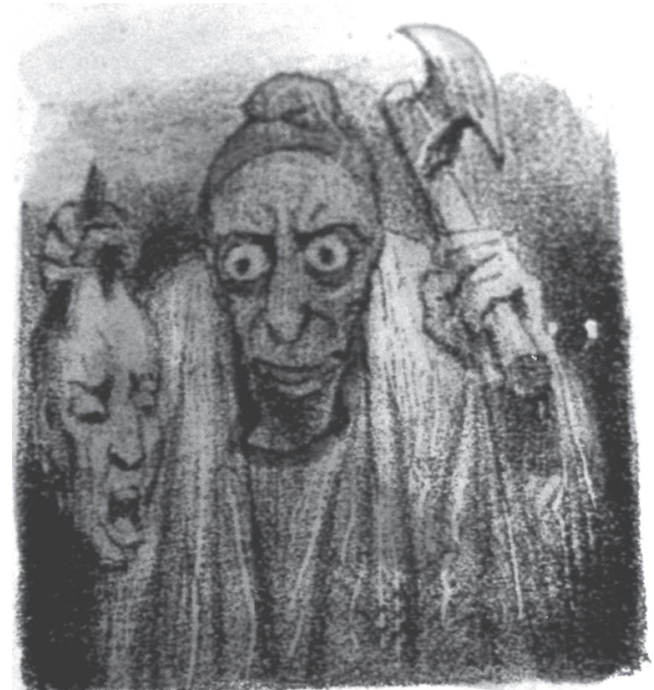

Figure 3. Carnaval politique, par Grandville et Forest, dans La Caricature, 10 mars 1831 (détail)

Habituant le regard du public à être confronté à de fréquents combats entre une femme qui figure autant la France que la Liberté (donc la République) et le roi les artistes de l'équipe de Philipon introduisent l'idée d'un combat à mort entre les deux, comme en témoigne une lithographie de Desperret, sans titre (figure 4) publiée dans le numéro du 2 juin 1831. Rappelant les promesses de Juillet 1830, la forme de la femme-liberté qui s'apprête à poignarder le roi reproduit presque exactement l'ombre d'un Louis-Philippe hagard, fuyant le souvenir des Trois Glorieuses et de ses promesses ${ }^{13}$; il est intéressant de mettre l'inspiration de la caricature de Desperret en relation avec le célèbre tableau de Pierre-Paul Prud'hon $\mathrm{La}$ Justice et la Vengeance divine poursuivant le Crime, exposé avec un très grand succès en 1808. La création de cette ombre vengeresse rappelle au moins deux choses: tout d'abord, que les dessinateurs ont été nourris de culture

\footnotetext{
${ }^{12}$ Le carnaval politique de Grandville et Forest, publié dans La caricature, n¹6 en date du 17 février puis du n 19 en date du 10 mars1831.

${ }^{13}$ On nomme « les Trois Glorieuses » les journées révolutionnaires des 27, 28 et 29 juillet 1830.
} 


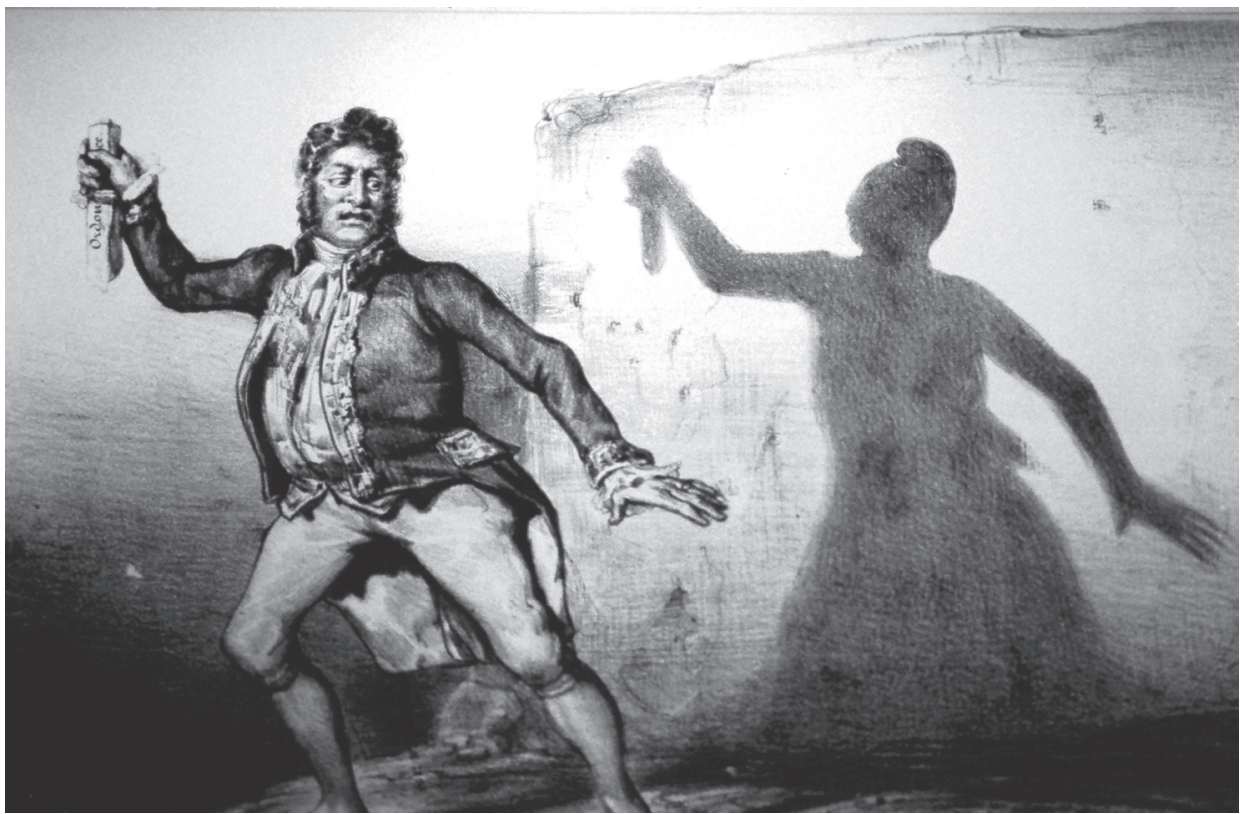

Figure 4. Sans titre, par Desperret, dans La Caricature, 2 juin 1831

classique et, ensuite, que le vocabulaire des signes n'est pas extensible à l'infini.

Un autre système de gouvernement peut-il être possible pour que Chacun son tour en politique, comme l'indique le titre d'une lithographie de Grandville et Forest publiée le 27 décembre 1833? Auparavant, il faudra bien trier le bon grain de l'ivraie, et peut-être même subir une nouvelle passion comme en témoigne cette Nouvelle cène (figure 5), de Bouquet publiée le 17 mai 1832. La femme-liberté qui a pris la place du Christ prononce aussi ses propres paroles, «en vérité, en vérité je vous le dis, il en est un parmi nous qui me trahira».

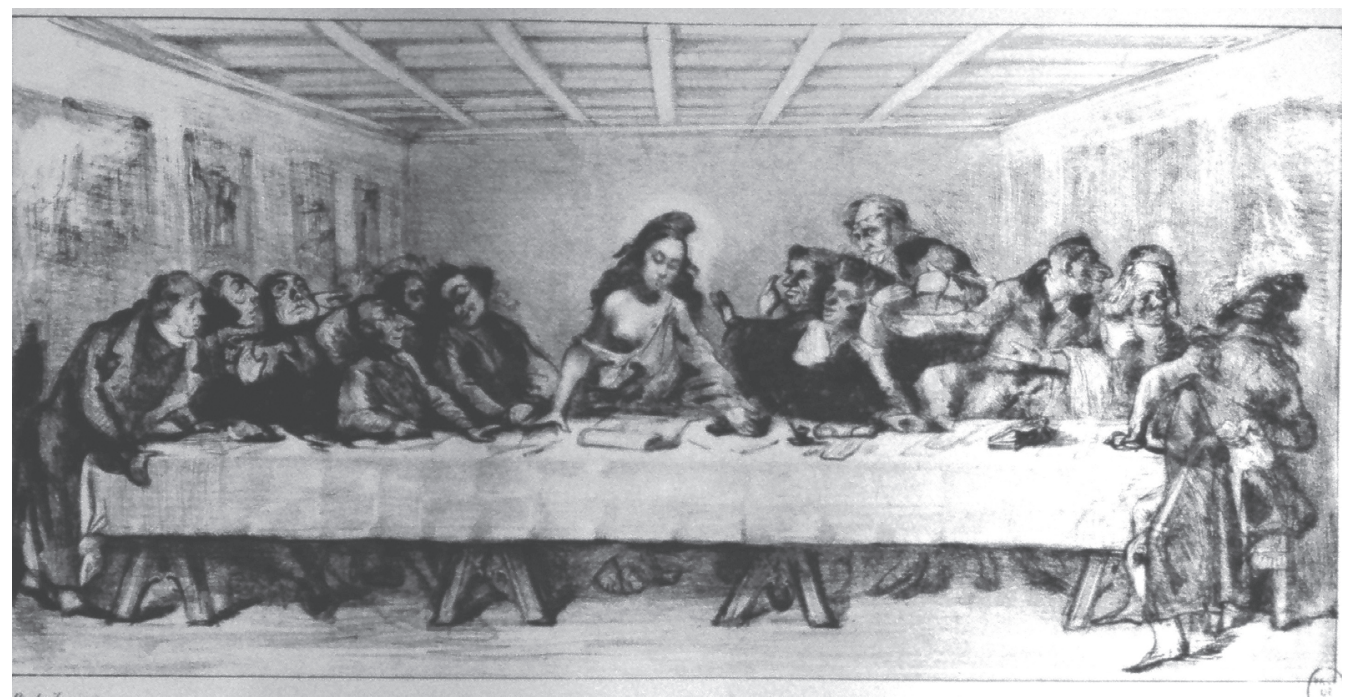

Figure 5. Nouvelle cène, par Bouquet, dans La Caricature, 17 mai 1832 
Après les références antiques et les références religieuses, les auteurs de caricatures trouvent aussi leur inspiration dans le jeu, comme en témoigne cette partie d'échecs anonyme en date du 11 oct 1832 , Quand finira cette partie? (figure 6). LouisPhilippe, face à une femme-liberté à bonnet phrygien qui semble gagner la partie est aussi démuni que pouvait se trouver son ancêtre Louis XVI en 1792 face à un sans-culotte sur une gravure judicieusement intitulée
Repique est Capet ${ }^{14}$. La phrase, «quand finira cette partie?» est très ambivalente, et peut être placée dans la bouche de chacun des deux personnages, soit en signe d'espérance soit en signe de lassitude. Toutefois, un examen exhaustif des dessins du journal $L a$ Caricature montre que la présence d'une femme à bonnet phrygien ne renvoie pas uniquement à l'idée de «République», mais très souvent à l'idée de liberté en général, liberté de la presse en particulier ${ }^{15}$.

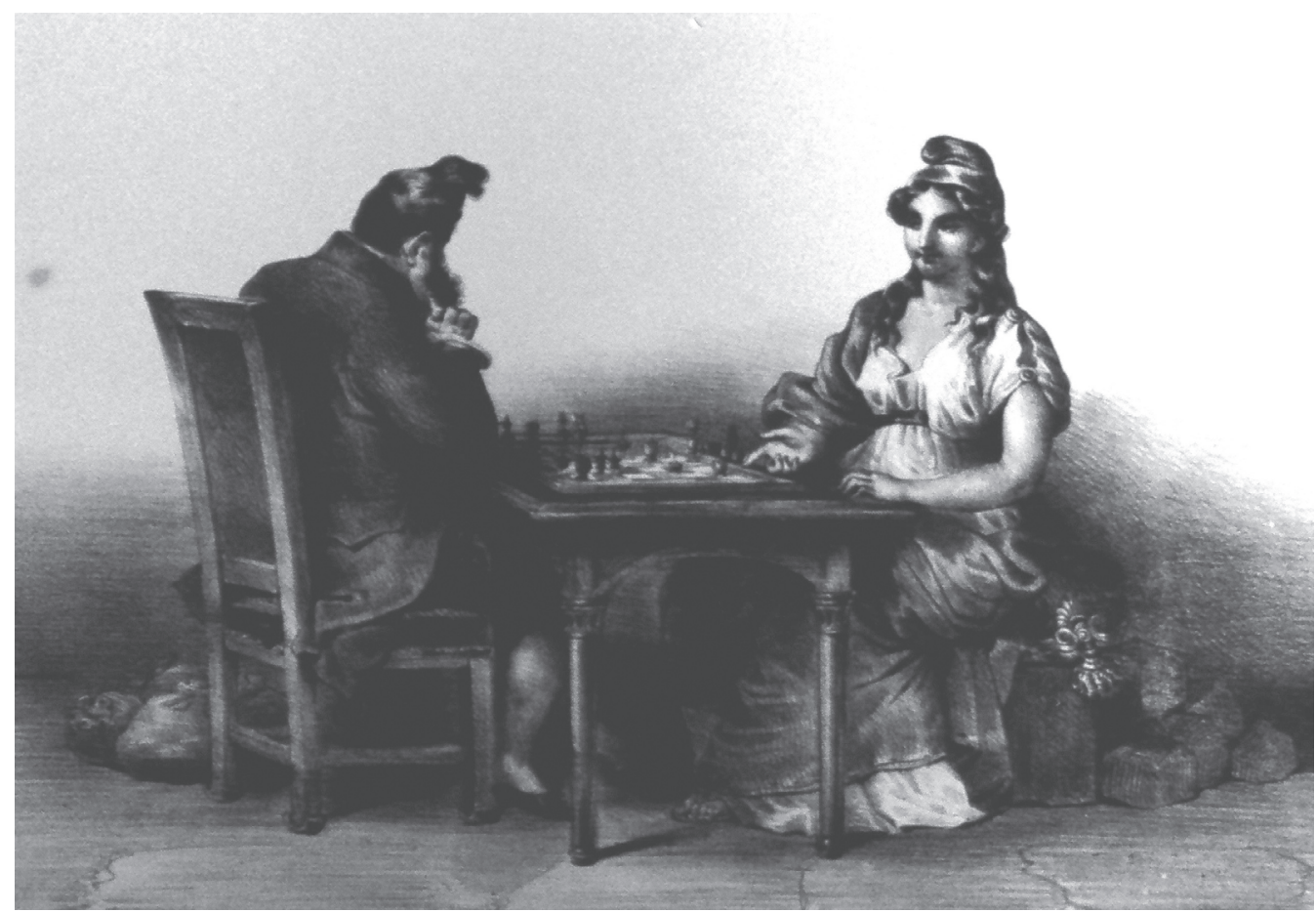

Figure 6. Quand finira cette partie? anonyme, dans La Caricature, 11 octobre 1832

\footnotetext{
${ }^{14}$ Annie Duprat, Le roi décapité. Essais sur les imaginaires politiques, Paris, Cerf, 1992, p. 188-199.

${ }^{15}$ Voir la lithographie Parodie du tableau de Proudhon : la justice et la vengeance divines poursuivant le crime, publiée le 13 février 1834 ; Machine infernale de Sauzet, du 20 août 1835. Entre 1830 et 1835, le dessinateur Decamps a décliné les vies multiples d'une «Françoise » presque devenue « Marianne ». Il la figure comme une jeune femme, le plus souvent enchaînée, mais aussi petite fille tenue en laisse le 5 mars 1831 sous le titre Françoise-Désirée-Liberté, née à Paris le 27 juillet 1830, ou, successivement, attachée au poteau d'exécution sur un Jugement de Françoise Liberté, le 27 janvier 1831, puis crucifiée sur une Exécution de Désirée-Françoise-Libertéle 12 juin 1834 ; elle est trahie au Mont des Oliviers sur Agonie de la Liberté au jardin des Oliviers de Bouquet, le 2 août 1832 ; mais elle a aussi remplacé le Christ sur des cènes renouvelées comme La Cène de Bouquet, le 17 mai 1832 ou sur la Scène révolutionnaire (sic) de Benjamin, le 25 décembre 1834.
} 
Au milieu des années 1830, le transfert d'une allégorie antiquisante et un brin pompeuse, la Liberté, vers une figure plus familière, proche de la Marianne de la IIIè République, a été opéré par l'art de la caricature grâce à des mises en scène et des mises en images permettant sa fusion avec la représentation de la République. Le langage de l'allégorie est alors devenu incompréhensible aux contemporains, comme le montre la réception du tableau de Delacroix, La Liberté guidant le peuple, dans lequel celui-ci, justement, reconnaît une «République», une "Marianne sur les barricades» que le peintre n'avait certainement pas voulu peindre ${ }^{16}$. La généalogie iconographique peut s'écrire comme le passage insensible de la «Liberté» à la «République», puis, dans les années 1850 , la personnification de la République sous le nom de Marianne. Avec la Commune de Paris et les débuts difficiles de la Illè République, plusieurs Mariannes s'affrontent, de la vamp à la concierge ou de la République radicale à la République modérée, ces métamorphoses de Marianne sont bien connues ${ }^{17}$. Marianne, enfin se confond avec la France, comme en témoignent les étrangers lorsqu'ils évoquent notre pays ${ }^{18}$.

\footnotetext{
${ }^{16}$ Sur l'identité de la femme sur les barricades, voir Annie Duprat, « Des femmes sur les barricades de juillet 1830 : histoire d'un imaginaire social », La barricade, Paris, Publications de la Sorbonne, 1997, p. 197-208.

${ }^{17}$ Maurice Agulhon et Pierre Bonte, Marianne, les visages de la République, Paris, Gallimard, collection Découvertes, 1992 ; JeanMichel Renault, Les fées de la République, Paris, Pélican/Vilo, 2003.

${ }^{18}$ Voir l'article de Nina Bernstein «Marianne, cette femme fatale qui agace Bush le macho » publié dans le New York Times en octobre 2003.
} 